\title{
NONEXISTENCE OF A CIRCULANT EXPANDER FAMILY
}

\author{
KA HIN LEUNG, VINH NGUYEN and WASIN SO ${ }^{凶}$
}

(Received 5 February 2010)

\section{Abstract}

The expansion constant of a simple graph $G$ of order $n$ is defined as

$$
h(G)=\min _{0<|S| \leq n / 2} \frac{|E(S, \bar{S})|}{|S|}
$$

where $E(S, \bar{S})$ denotes the set of edges in $G$ between the vertex subset $S$ and its complement $\bar{S}$. An expander family is a sequence $\left\{G_{i}\right\}$ of $d$-regular graphs of increasing order such that $h\left(G_{i}\right)>\epsilon$ for some fixed $\epsilon>0$. Existence of such families is known in the literature, but explicit construction is nontrivial. A folklore theorem states that there is no expander family of circulant graphs only. In this note, we provide an elementary proof of this fact by first estimating the second largest eigenvalue of a circulant graph, and then employing Cheeger's inequalities

$$
\frac{d-\lambda_{2}(G)}{2} \leq h(G) \leq \sqrt{2 d\left(d-\lambda_{2}(G)\right)}
$$

where $G$ is a $d$-regular graph and $\lambda_{2}(G)$ denotes the second largest eigenvalue of $G$. Moreover, the associated equality cases are discussed.

2000 Mathematics subject classification: primary 05C50.

Keywords and phrases: circulant graph, Cheeger's inequality, expander family.

\section{Circulant graphs}

Let $G$ be a simple graph and let $V(G)$ denote its vertex set. Then $G$ is said to have order $n$ if $|V(G)|=n$. The spectrum $\operatorname{Sp}(G)$ of $G$ is the collection of eigenvalues of the adjacency matrix $A(G)$ of $G$. Since $A(G)$ is a real symmetric matrix, all its eigenvalues are real and they are denoted by $\lambda_{1}(G) \geq \lambda_{2}(G) \geq \cdots \geq \lambda_{n}(G)$. Furthermore, $G$ is $d$-regular if every vertex has degree $d$.

Definition 1.1. A graph is circulant if it has a circulant adjacency matrix.

Circulant graphs are special regular graphs whose spectra can be computed explicitly in terms of their symbols.

(C) 2010 Australian Mathematical Publishing Association Inc. 0004-9727/2010 \$16.00 
Definition 1.2. A subset $S$ of $\{1,2, \ldots, n-1\}$ is called a symbol of a circulant graph $G$ of order $n$ if $A(G)=\sum_{r \in S} Z^{r}$, where $Z$ is the $n \times n$ matrix

$$
\left[\begin{array}{ccccc}
0 & 1 & 0 & \ldots & 0 \\
0 & 0 & 1 & \ldots & 0 \\
0 & 0 & 0 & \ldots & 0 \\
\vdots & \vdots & \vdots & \ddots & \vdots \\
1 & 0 & 0 & \ldots & 0
\end{array}\right] .
$$

If $S$ is a symbol of a $d$-regular circulant graph of order $n$, then $|S|=d$ and $r \in S$ if and only if $n-r \in S$. Note that the characteristic polynomial of $Z$ is $x^{n}-1$ and so its eigenvalues are $1, \omega, \omega^{2}, \ldots, \omega^{n-1}$ where $\omega=e^{2 \pi \mathbf{i} / n}$ and $\mathbf{i}=\sqrt{-1}$. For $1 \leq k \leq n$,

$$
\sum_{r \in S} \omega^{r k}=\sum_{r \in S} \omega^{(n-r) k}=\sum_{r \in S} \omega^{-r k}
$$

and so

$$
\sum_{r \in S} \omega^{r k}=\sum_{r \in S} \cos (2 \pi r k / n) .
$$

Hence the spectrum of a circulant graph can be explicitly described by its symbol as follows.

THEOREM 1.3. Let $S$ be a symbol of a d-regular circulant graph $G$ of order $n$. Then the spectrum of $G$ is

$$
\operatorname{Sp}(G)=\left\{\sum_{r \in S} \cos (2 \pi r k / n): 1 \leq k \leq n\right\} .
$$

The next theorem is a special case of Dirichlet's theorem in the literature on simultaneous Diophantine approximations [8, Section 8.2]. For the sake of completeness, we include its proof.

THEOREM 1.4. Given real numbers $\alpha_{1}, \ldots, \alpha_{t}$ and a positive integer $q$, there exist integers $k, x_{1}, \ldots, x_{t}$ such that $1 \leq k \leq q^{t}$ and $\left|\alpha_{i} k-x_{i}\right| \leq 1 / q$ for all $i=$ $1,2, \ldots, t$.

PROOF. Consider the $q^{t}+1$ points

$$
\left\{\left(u \alpha_{1}-\left\lfloor u \alpha_{1}\right\rfloor, \ldots, u \alpha_{t}-\left\lfloor u \alpha_{t}\right\rfloor\right): 0 \leq u \leq q^{t}\right\}
$$

in the $t$-dimensional unit cube $[0,1)^{t}$. Partition $[0,1)^{t}$ into $q^{t}$ disjoint compartments

$$
\left\{\left[\frac{j_{1}}{q}, \frac{j_{1}+1}{q}\right) \times \cdots \times\left[\frac{j_{t}}{q}, \frac{j_{t}+1}{q}\right): 0 \leq j_{1}, \ldots, j_{t} \leq q-1\right\} .
$$

By the pigeonhole principle, there exist two points $\left(u \alpha_{1}-\left\lfloor u \alpha_{1}\right\rfloor, \ldots, u \alpha_{t}-\left\lfloor u \alpha_{t}\right\rfloor\right)$ and $\left(v \alpha_{1}-\left\lfloor v \alpha_{1}\right\rfloor, \ldots, v \alpha_{t}-\left\lfloor v \alpha_{t}\right\rfloor\right)$ lying in the same compartment

$$
\left[\frac{j_{1}}{q}, \frac{j_{1}+1}{q}\right) \times \cdots \times\left[\frac{j_{t}}{q}, \frac{j_{t}+1}{q}\right) .
$$


Without loss of generality, we may suppose that $u>v$. Then, for $1 \leq i \leq t$,

$$
\left|(u-v) \alpha_{i}-\left(\left\lfloor u \alpha_{i}\right\rfloor-\left\lfloor v \alpha_{i}\right\rfloor\right)\right|=\left|\left(u \alpha_{i}-\left\lfloor u \alpha_{i}\right\rfloor\right)-\left(v \alpha_{i}-\left\lfloor v \alpha_{i}\right\rfloor\right)\right| \leq \frac{1}{q} .
$$

Hence we can take $k=u-v$ and $x_{i}=\left\lfloor u \alpha_{i}\right\rfloor-\left\lfloor v \alpha_{i}\right\rfloor$ as required.

We need the following corollary for our main result in Theorem 1.6.

COROLlARY 1.5. Given positive integers $r_{1}, \ldots, r_{t}$ and $n$, there exist integers $k, x_{1}$, $\ldots, x_{t}$ such that $1 \leq k \leq n-1$ and $\left|k r_{i} / n-x_{i}\right| \leq 1 /\left((n-1)^{1 / t}-1\right)$ for all $i$.

PROOF. Take $\alpha_{i}=r_{i} / n$ and $q=\left\lfloor(n-1)^{1 / t}\right\rfloor$. Then, by Theorem 1.4, there exist integers $k, x_{1}, \ldots, x_{t}$ such that

$$
1 \leq k \leq q^{t} \leq n-1
$$

and

$$
\left|k r_{i} / n-x_{i}\right|=\left|\alpha_{i} k-x_{i}\right| \leq 1 / q=1 /\left\lfloor(n-1)^{1 / t}\right\rfloor \leq 1 /\left((n-1)^{1 / t}-1\right) \quad \text { for all } i .
$$

This concludes the proof.

THEOREM 1.6. Let $G$ be a d-regular circulant graph of order $n$. Then

$$
\lambda_{2}(G) \geq d-\frac{2 \pi^{2} d}{\left((n-1)^{1 / d}-1\right)^{2}} .
$$

PROOF. Let a symbol of $G$ be $\left\{r_{1}, r_{2}, \ldots, r_{d}\right\}$. By Corollary 1.5 , there exist integers $k_{0}, x_{1}, \ldots, x_{d}$ such that $1 \leq k_{0} \leq n-1$ and $\left|k_{0} r_{i} / n-x_{i}\right| \leq 1 /\left((n-1)^{1 / d}-1\right)$. By Theorem 1.3,

$$
\lambda_{2}(G)=\max _{1 \leq k \leq n-1} \cos \left(2 \pi r_{1} k / n\right)+\cdots+\cos \left(2 \pi r_{d} k / n\right) .
$$

Consequently,

$$
\begin{aligned}
\lambda_{2}(G) & \geq \cos \frac{2 \pi r_{1} k_{0}}{n}+\cdots+\cos \frac{2 \pi r_{d} k_{0}}{n} \\
& =\cos 2 \pi\left(\frac{r_{1} k_{0}}{n}-x_{1}\right)+\cdots+\cos 2 \pi\left(\frac{r_{d} k_{0}}{n}-x_{d}\right) \\
& \geq\left(1-\frac{1}{2} 4 \pi^{2}\left|\frac{r_{1} k_{0}}{n}-x_{1}\right|^{2}\right)+\cdots+\left(1-\frac{1}{2} 4 \pi^{2}\left|\frac{r_{d} k_{0}}{n}-x_{d}\right|^{2}\right) \\
& =d-2 \pi^{2}\left(\left|\frac{r_{1} k_{0}}{n}-x_{1}\right|^{2}+\cdots+\left|\frac{r_{d} k_{0}}{n}-x_{d}\right|^{2}\right) \\
& \geq d-\frac{2 \pi^{2} d}{\left((n-1)^{1 / d}-1\right)^{2}},
\end{aligned}
$$

where the first equality and second inequality are justified by the fact that the cosine function has a period of $2 \pi$ and $\cos x \geq 1-\frac{1}{2} x^{2}$, respectively. 
There are better lower bounds of $\lambda_{2}(G)$ in the literature. For instance, Friedman et al. [4] used a sphere packing argument to show that

$$
\lambda_{2}(G) \geq d-\frac{c_{d} d}{n^{4 / d}}
$$

for some absolute constant $c_{d}$ and for any $d$-regular Cayley graph $G$ on an abelian group, including circulant graph. However, their proof is more involved and less elementary.

\section{Expansion constant}

Throughout this section, let $G$ denote a $d$-regular graph of order $n$. First we note that $\lambda_{1}(G)=d$, and that the difference $d-\lambda_{2}(G)$ between the first two largest eigenvalues of $G$ is an important quantity. Indeed, $d-\lambda_{2}(G)=0$ if and only if $G$ is disconnected. Moreover, $d-\lambda_{2}(G)$ is closely related to the expansion constant of $G$.

DEFINITION 2.1. The expansion constant of $G$ is defined as

$$
h(G)=\min _{0<|S| \leq n / 2} \frac{|E(S, \bar{S})|}{|S|}
$$

where $E(S, \bar{S})$ denotes the set of edges in $G$ between the vertex subset $S$ and its complement $\bar{S}$.

Note that $h(G) \geq 0$ and equality holds if and only if $G$ is disconnected. It is not hard to see that $h\left(K_{n}\right)=\lceil n / 2\rceil$ and $h\left(C_{n}\right)=2 /\lfloor n / 2\rfloor$ where $K_{n}$ is the complete graph of order $n$ and $C_{n}$ is the cycle graph of order $n$. In general, the expansion constant of $G$ is hard to compute directly [2], but it can be estimated through the following inequalities due to Cheeger. Although the proof of Cheeger's inequalities exists in the literature (see, for instance, [5]), we repeat it here in order to discuss the equality cases.

THEOREM 2.2.

$$
\frac{d-\lambda_{2}(G)}{2} \leq h(G) \leq \sqrt{2 d\left(d-\lambda_{2}(G)\right)} .
$$

The right equality holds if and only if $G$ is disconnected. The left equality holds if and only if $G$ is disconnected or $G$ has a vertex set $S$ such that both induced subgraphs $G_{S}$ and $G_{\bar{S}}$ are $k$-regular graphs of same order and $\lambda_{2}(G)=2 k-d$.

PROOF. (i) We first prove the right inequality:

$$
h(G) \leq \sqrt{2 d\left(d-\lambda_{2}(G)\right)}
$$

or, equivalently, $h(G)^{2} / 2 d \leq d-\lambda_{2}(G)$.

Let $g=\left[g_{1}, \ldots, g_{n}\right]^{T}$ be an eigenvector of $A=A(G)$ corresponding to $\lambda_{2}=$ $\lambda_{2}(G)$. Since $g$ is orthogonal to the column vector of all ones, the $g_{i}$ s are not all of the same sign. By relabeling, and replacing $g$ by $-g$ if necessary, we can assume that

$$
g_{1} \geq \cdots \geq g_{r}>0 \geq g_{r+1} \geq \cdots \geq g_{n} \quad \text { where } 1 \leq r \leq n / 2 .
$$


Write $g=\left[g_{+}-g_{-}\right]^{T}$ and $f=\left[g_{+} 0\right]^{T}$ where $g_{+}$is a positive vector and $g_{-}$is a nonnegative vector. Note that $f$ is a nonzero vector with at least one zero entry, and

$$
f_{1} \geq \cdots \geq f_{r}>0=f_{r+1}=\cdots=f_{n} .
$$

Partition $A=\left[\begin{array}{cc}B & X \\ X^{T} & C\end{array}\right]$ where $B$ is an $r \times r$ matrix. Let $L=d I-A$ where $I$ is the $n \times n$ identity matrix. To prove the right inequality, it suffices to show two auxiliary inequalities. The first is $f^{T} L f / f^{T} f \leq d-\lambda_{2}$. To this end, we note that $A g=\lambda_{2} g$ gives $\lambda_{2} g_{+}=B g_{+}-X g_{-} \leq B g_{+}$because $X$ and $g_{-}$are nonnegative. Hence

$$
\lambda_{2} f^{T} f=\lambda_{2} g_{+}^{T} g_{+} \leq g_{+}^{T} B g_{+}=f^{T} A f
$$

which is equivalent to $f^{T} L f / f^{T} f \leq d-\lambda_{2}$.

The second is $h(G)^{2} / 2 d \leq f^{T} L f / f^{T} f$. To prove this we consider

$$
\begin{array}{rl}
B_{f}= & \sum_{(x<y) \in E(G)}\left|f_{x}^{2}-f_{y}^{2}\right| \\
= & \sum_{(x<y) \in E(G)}\left(f_{x}^{2}-f_{y}^{2}\right) \quad \text { because the } f_{i} \text { s are decreasing } \\
= & \sum_{(x<y) \in E(G)} \sum_{i=x}^{y-1}\left(f_{i}^{2}-f_{i+1}^{2}\right) \quad \text { (telescopic sum) } \\
= & |\{(x<y): x \leq 1, y \geq 2\}|\left(f_{1}^{2}-f_{2}^{2}\right) \\
& \quad+|\{(x<y): x \leq 2, y \geq 3\}|\left(f_{2}^{2}-f_{3}^{2}\right)+\cdots \\
= & |E([1], \overline{[1]})|\left(f_{1}^{2}-f_{2}^{2}\right)+|E([2], \overline{[2]})|\left(f_{2}^{2}-f_{3}^{2}\right)+\cdots \\
& \quad+|E([n-1], \overline{[n-1]})|\left(f_{n-1}^{2}-f_{n}^{2}\right) \\
= & \sum_{i=1}^{n-1}|E([i], \overline{[i]})|\left(f_{i}^{2}-f_{i+1}^{2}\right) \\
= & h(G)\left(f_{1}^{2}+\cdots+f_{n}^{2}\right) \quad \text { because } f_{r+1}=\cdots=f_{n}=0 \\
= & \sum_{i=1}^{r}|E([i], \overline{[i]})|\left(f_{i}^{2}-f_{i+1}^{2}\right) \quad \text { because } f_{r+1}=\cdots=f_{n}=0 \\
r & h(G)\left(f^{T} f\right) . \\
& \\
& \\
&
\end{array}
$$


On the other hand,

$$
\begin{aligned}
B_{f} & =\sum_{(x<y) \in E(G)}\left|f_{x}^{2}-f_{y}^{2}\right| \\
& =\sum_{(x<y) \in E(G)}\left|f_{x}+f_{y}\right|\left|f_{x}-f_{y}\right| \\
& \leq\left(\sum_{(x<y) \in E(G)}\left|f_{x}+f_{y}\right|^{2}\right)^{1 / 2}\left(\sum_{(x<y) \in E(G)}\left|f_{x}-f_{y}\right|^{2}\right)^{1 / 2} \text { by Cauchy-Schwarz } \\
& \leq\left(\sum_{(x<y) \in E(G)} 2\left(f_{x}^{2}+f_{y}^{2}\right)\right)^{1 / 2}\left(\sum_{(x<y) \in E(G)}\left|f_{x}-f_{y}\right|^{2}\right)^{1 / 2} \\
& =\left(2 d\left(f^{T} f\right)\right)^{1 / 2}\left(\sum_{(x<y) \in E(G)}\left|f_{x}-f_{y}\right|^{2}\right)^{1 / 2} \text { because } G \text { is } d \text {-regular } \\
& =\sqrt{2 d\left(f^{T} f\right)\left(f^{T} L f\right) .}
\end{aligned}
$$

Hence

$$
h(G)\left(f^{T} f\right) \leq B_{f} \leq \sqrt{2 d\left(f^{T} f\right)\left(f^{T} L f\right)},
$$

which is equivalent to $h(G)^{2} / 2 d \leq f^{T} L f / f^{T} f$.

(ii) We next address the right equality, $h(G)^{2} / 2 d=d-\lambda_{2}(G)$. If $G$ is disconnected then $h(G)=d-\lambda_{2}(G)=0$, hence the right equality holds. On the other hand, if $G$ is connected and the right equality holds then $h(G)^{2} / 2 d=f^{T} L f / f^{T} f$. From the proof above, it follows that $B_{f}=\sqrt{2 d\left(f^{T} L f\right)\left(f^{T} f\right)}$. Hence, $\left(f_{x}+f_{y}\right)^{2}=$ $2\left(f_{x}^{2}+f_{y}^{2}\right)$; that is, $f_{x}=f_{y}$ for all $(x<y) \in E(G)$. Thus $f$ is a constant vector because $G$ is a connected graph. This is impossible since $f$ is a nonzero vector with at least one zero entry.

(iii) We now prove the left inequality, $\lambda_{2} \geq d-2 h(G)$. Let $S_{0} \subseteq V(G)$ such that $h(G)=\left|E\left(S_{0}, \overline{S_{0}}\right)\right| /\left|S_{0}\right|$ and $\left|S_{0}\right| \leq n / 2$. Relabel $G$ with vertex set $S_{0}$ first and then $\overline{S_{0}}$, and partition $A(G)$ as $\left[\begin{array}{cc}B & X \\ X^{T} & C\end{array}\right]$ accordingly. Let

$$
f_{0}=\left|\overline{S_{0}}\right|\left[\begin{array}{c}
\mathbf{e}_{S_{0}} \\
\mathbf{0}
\end{array}\right]-\left|S_{0}\right|\left[\begin{array}{c}
\mathbf{0} \\
\mathbf{e}_{\overline{S_{0}}}
\end{array}\right],
$$

where $\mathbf{e}_{S_{0}}$ and $\mathbf{e}_{\overline{S_{0}}}$ are the column vectors with all ones of lengths $\left|S_{0}\right|$ and $\left|\overline{S_{0}}\right|$, respectively. Note that $f_{0}$ is orthogonal to $\mathbf{e}=\left[\begin{array}{c}\mathbf{e}_{S_{0}} \\ \mathbf{e}_{S_{0}}\end{array}\right]$ and $f_{0}^{T} f_{0}=n\left|S_{0}\right|\left|\overline{S_{0}}\right|$. Moreover, using the regularity of $G$,

$$
\mathbf{e}_{S_{0}}^{T} B \mathbf{e}_{S_{0}}=d\left|S_{0}\right|-\left|E\left(S_{0}, \overline{S_{0}}\right)\right|, \quad \mathbf{e}_{\overline{S_{0}}}^{T} C \mathbf{e}_{\overline{S_{0}}}=d\left|\overline{S_{0}}\right|-\left|E\left(S_{0}, \overline{S_{0}}\right)\right|,
$$

and

$$
\mathbf{e}_{S_{0}}^{T} X \mathbf{e}_{\overline{S_{0}}}=\mathbf{e}_{\overline{S_{0}}}^{T} X^{T} \mathbf{e}_{S_{0}}=\left|E\left(S_{0}, \overline{S_{0}}\right)\right|
$$


Hence $f_{0}^{T} A f_{0}=\left|S_{0}\right|\left|\overline{S_{0}}\right| d n-\left|E\left(S_{0}, \overline{S_{0}}\right)\right| n^{2}$. Consequently,

$$
\begin{aligned}
\lambda_{2} & =\max _{f \perp \mathbf{e}} \frac{f^{T} A f}{f^{T} f} \\
& \geq \frac{f_{0}^{T} A f_{0}}{f_{0}^{T} f_{0}} \quad \text { because } f_{0} \perp \mathbf{e} \\
& =\frac{\left|S_{0}\right|\left|\overline{S_{0}}\right| d n-\left|E\left(S_{0}, \overline{S_{0}}\right)\right| n^{2}}{n\left|S_{0}\right|\left|\overline{S_{0}}\right|} \\
& =d-\frac{n}{\left|\overline{S_{0}}\right|} \frac{\left|E\left(S_{0}, \overline{S_{0}}\right)\right|}{\left|S_{0}\right|} \\
& =d-\frac{n}{\left|\overline{S_{0}}\right|} h(G) \quad \text { by the choice of } S_{0} \\
& \geq d-2 h(G) \quad \text { by the choice of } S_{0} .
\end{aligned}
$$

(iv) We conclude with the left equality, $\frac{1}{2}\left(d-\lambda_{2}(G)\right)=h(G)$. If $G$ is disconnected then $\lambda_{2}(G)=d$ and $h(G)=0$, hence $\frac{1}{2}\left(d-\lambda_{2}(G)\right)=h(G)$. If the $d$-regular graph $G$ has a vertex subset $|S|=n / 2$ such that $G_{S}$ and $G_{\bar{S}}$ are both $k$-regular and $2 k-d=$ $\lambda_{2}(G)$, then $h(G)=\frac{1}{2}\left(d-\lambda_{2}(G)\right)$. In any case, the left equality holds.

On the other hand, if the left equality holds and $G$ is connected then $\left|S_{0}\right|=n / 2$ because $h(G)>0$, and $f_{0}$ is an eigenvector of $A$ corresponding to $\lambda_{2}$. It follows that $\left|S_{0}\right|=n / 2, B \mathbf{e}_{S_{0}}=\frac{1}{2}\left(\lambda_{2}+d\right) \mathbf{e}_{S_{0}}$ and $C \mathbf{e}_{\overline{S_{0}}}=\frac{1}{2}\left(\lambda_{2}+d\right) \mathbf{e}_{\overline{S_{0}}}$; that is, $k=\left(\lambda_{2}+d\right) / 2$ is an integer, and both induced subgraphs $G_{S_{0}}$ and $G_{\overline{S_{0}}}$ are $k$-regular.

There are many graphs achieving the left equality. Here we include a few examples.

Example 2.3. Let $P$ be the Petersen graph. Then $d=3, \lambda_{2}(G)=1$ and $h(G)=1$. Hence the left equality holds. Moreover, take $S_{0}$ such that $G_{S_{0}}$ and $G_{\overline{S_{0}}}$ are the outer and inner 5-cycles, respectively, which are 2-regular of order five.

EXAMPLE 2.4. Let $G=K_{2 r, 2 r, \ldots, 2 r}$ be the $2 r(t-1)$-regular complete $t$-partite graph with $t>1$. Then $d=2 r(t-1), \lambda_{2}(G)=0$ and $h(G)=r(t-1)$. Hence the left equality holds. Moreover, there exists $S_{0}$ such that $G_{S_{0}}=K_{r, r, \ldots, r}$ and $G_{\overline{S_{0}}}=K_{r, r, \ldots, r}$ are $r(t-1)$-regular graphs of order $r t$.

EXAMPLE 2.5. Let $H$ be an $r$-regular graph with $\lambda_{2}(H) \leq r-2$. Then $G=H \times K_{2}$ is an $(r+1)$-regular graph with $d=r+1, \lambda_{2}(G)=r-1$ and $h(G)=1$. Hence the left equality holds. Moreover, take $S_{0}$ such that $G_{S_{0}}=H$ and $G_{\overline{S_{0}}}=H$ are $r$-regular graphs of the same order. In particular, starting with $H=K_{2}$ and repeating the above process $n-1$ times, we obtain $G=Q_{n}$, the hypercube, achieving the left equality.

EXAMPLE 2.6. Let $G_{1}$ and $G_{2}$ be the two nonisomorphic 3-regular graphs of order six. Let $G$ be the graph obtained by connecting the vertices of $G_{1}$ and $G_{2}$ by six independent edges, so that $G$ is a 4-regular graph of order 12. Now $\lambda_{2}(G)=2$ 
and $h(G)=1$. Hence the left equality holds. Moreover, take $S_{0}$ such that $G_{S_{0}}=G_{1}$ and $G_{\overline{S_{0}}}=G_{2}$ are 3-regular graphs of order six.

It would be nice to find a characterization of the left equality without referring to the eigenvalue of the graph.

\section{Nonexistence}

In this section, we provide a proof for the folklore theorem that there is no expander family of circulant graphs only. First we give the definition of an expander family.

DEFINITION 3.1. A family $\left\{G_{i}\right\}$ of $d$-regular graphs is an expander family if $\left|V\left(G_{i}\right)\right| \rightarrow \infty$ and $h\left(G_{i}\right)>\epsilon$ for some $\epsilon>0$.

If $\left\{G_{i}\right\}$ is an expander family then $G_{i}$ is connected for all $i$ because $h\left(G_{i}\right)>0$ and $d \geq 3$; otherwise we have $d=2$ then $G_{i}=C_{n_{i}}$ and so

$$
h\left(G_{i}\right)=h\left(C_{n_{i}}\right)=\frac{2}{\left\lfloor\frac{n_{i}}{2}\right\rfloor} \rightarrow 0,
$$

a contradiction. Expander families share many of the properties of random regular graphs, and their applications are discussed in [5]. The existence of expander families is known, and Pinsker [7] was the first to show the existence of expander families by a probabilistic method. The explicit construction of an expander family is nontrivial when $d$ and $\epsilon$ are prescribed. Maglius [6] was the first to construct an expander family. Cheeger's inequalities are of great help in studying expander families. Upper bounds for $\lambda_{2}(G)$ give lower bounds for $d-\lambda_{2}(G)$, and so provide a tool to construct expander families. On the other hand, lower bounds of $\lambda_{2}(G)$ give upper bounds of $d-\lambda_{2}(G)$, and so provide a tool to show the nonexistence of an expander family.

THEOREM 3.2. There is no circulant expander family.

PROOF. Suppose that there is a $d$-regular circulant expander family $\left\{G_{i}\right\}$; that is, there is an $\epsilon>0$ such that $h\left(G_{i}\right)>\epsilon$ for all $i$. Now, by Theorem 1.6, $d-\lambda_{2}\left(G_{i}\right) \leq$ $2 \pi^{2} d /\left(\left(n_{i}-1\right)^{1 / d}-1\right)^{2}$. Consequently, by the right inequality in Theorem 2.2,

$$
h\left(G_{i}\right) \leq \sqrt{2 d\left(d-\lambda_{2}\left(G_{i}\right)\right)} \leq \sqrt{\frac{4 \pi^{2} d^{2}}{\left(\left(n_{i}-1\right)^{1 / d}-1\right)^{2}}}=\frac{2 \pi d}{\left(n_{i}-1\right)^{1 / d}-1}
$$

where $n_{i}=\left|V\left(G_{i}\right)\right|$. Since $n_{i} \rightarrow \infty$, we have $h\left(G_{i}\right) \rightarrow 0$, which is a contradiction.

Friedman et al. [4] proved a stronger result that there is no expander family of $d$-regular Cayley graphs on abelian groups. Cioaba [3] provided yet another proof of this stronger result. Nonetheless our proof seems to be simpler and more elementary.

\section{Acknowledgement}

The authors would like to thank Professor Sebastian Cioaba for bringing to our attention references [1, 3, 4]. 


\section{References}

[1] N. Alon and Y. Roichman, 'Random Cayley graphs and expanders', Random Structures Algorithms 5 (1994), 271-284.

[2] M. Blum, R. M. Karp, O. Vornberger, C. H. Papadimitriou and M. Yannakakis, 'The complexity of testing whether a graph is a superconcentrator', Inform. Process. Lett. 13 (1981), 164-167.

[3] S. Cioaba, 'Closed walks and eigenvalues of Abelian Cayley graphs', C. R. Acad. Sci. Paris, Sér. I 342 (2006), 635-638.

[4] J. Friedman, R. Murty and J.-P. Tillich, 'Spectral estimates for Abelian Cayley graphs', J. Combin. Theory Ser. B 96 (2006), 111-121.

[5] S. Hoory, N. Linial and A. Wigderson, 'Expander graphs and their applications', Bull. Amer. Math. Soc. 43 (2006), 439-561.

[6] G. A. Margulis, 'Explicit constructions of concentrators', Probl. Inf. Transm. 9 (1973), 325-332.

[7] M. S. Pinsker, 'On the complexity of a concentrator', in: Proceedings, 7th International Teletraffic Conference, Stockholm, 1973, pp. 318/1-318/4.

[8] E. C. Titchmarsh, The Theory of the Riemann Zeta-function (Oxford University Press, Oxford, 1951).

KA HIN LEUNG, Department of Mathematics, National Singapore University, Singapore 119260, Republic of Singapore

e-mail: matlkh@nus.edu.sg

VINH NGUYEN, Department of Mathematics, San Jose State University, San Jose, CA 95192-0103, USA

e-mail: hacbao@gmail.com

WASIN SO, Department of Mathematics, San Jose State University, San Jose, CA 95192-0103, USA

e-mail: so@math.sjsu.edu 